\section{(6) OPEN ACCESS}

RESEARCH PAPER

\title{
Cognitive domain deficits in patients with aneurysmal subarachnoid haemorrhage at 1 year
}

\author{
George Kwok Chu Wong, ${ }^{1}$ Sandy Wai Lam, ${ }^{1}$ Karine Ngai, ${ }^{1}$ Adrian Wong, ${ }^{2}$ \\ Deyond $\mathrm{Siu}_{1}{ }^{3}$ Wai Sang Poon, ${ }^{1}$ Vincent Mok, ${ }^{2}$ Cognitive Dysfunction after Aneurysmal \\ Subarachnoid Hemorrhage Investigators
}

\begin{abstract}
${ }^{1}$ Division of Neurosurgery, Department of Surgery, Prince of Wales Hospital, The Chinese University of Hong Kong, Shatin, New Territories, Hong Kong

${ }^{2}$ Division of Neurology, Department of Medicine and Therapeutics, Prince of Wales Hospital, The Chinese University of Hong Kong, Shatin, New Territories, Hong Kong

${ }^{3}$ Department of Imaging and Interventional Radiology, Prince of Wales Hospital, The Chinese University of Hong Kong, Shatin, New Territories, Hong Kong

${ }^{4}$ Department of Psychological Studies, The Hong Kong Institute of Education, Tai Po, New Territories, Hong Kong
\end{abstract}

Correspondence to Dr George Kwok Chu Wong, Division of Neurosurgery, Department of Surgery, The Chinese University of Hong Kong, Prince of Wales Hospital, 4/F Clinical Science Building, 30-32 Ngan Shing Street, Shatin, New Territories, Hong Kong;

georgewong@surgery.cuhk. edu.hk

Received 5 November 2012 Revised 11 March 2013 Accepted 20 March 2013 Published Online First 20 April 2013

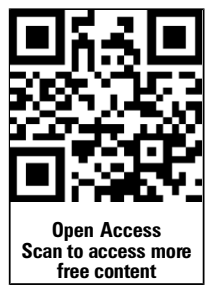

To cite: Wong GKC, Lam SW, Ngai K, et al. J Neurol Neurosurg Psychiatry 2013;84:1054-1058.

\section{ABSTRACT}

Background Cognitive domain deficits can occur after aneurysmal subarachnoid haemorrhage (aSAH) though few studies systemically evaluate its impact on 1-year outcomes.

Objective We aimed to evaluate the pattern and functional outcome impact of cognitive domain deficits in aSAH patients at 1 year.

Methods We carried out a prospective observational study in Hong Kong, during which, 168 aSAH patients (aged 21-75 years and had been admitted within $96 \mathrm{~h}$ of ictus) were recruited over a 26-month period. The cognitive function was assessed by a domain-specific neuropsychological assessment battery at 1 year after ictus. The current study is registered at ClinicalTrials.gov of the US National Institutes of Health (NCT01038193). Results Prevalence of individual domain deficits varied between $7 \%$ to $15 \%$, and $13 \%$ had two or more domain deficits. After adjusting for abbreviated National Institute of Health Stroke Scale and Geriatric Depressive Scale scores, unfavourable outcome (Modified Rankin Scale 3-5) and dependent instrumental activity of daily living (Lawton Instrumental Activity of Daily Living $<15$ ) were significantly associated with two or more domain deficits and number of cognitive domain deficits at 1 year. Two or more domain deficits was independently associated with age $(\mathrm{OR}, 1.1 ; 95 \% \mathrm{Cl} 1.1$ to 1.2 ; $\mathrm{p}<0.001)$ and delayed cerebral infarction $(O R, 6.1 ; 95 \%$ Cl 1.1 to $33.5 ; p=0.036)$, after adjustment for years of school education.

Interpretation In patients with aSAH, cognitive domain deficits worsened functional outcomes at 1 year. Delayed cerebral infarction was an independent risk factor for two or more domain deficits at 1 year.

\section{INTRODUCTION}

Although aneurysmal subarachnoid haemorrhage (aSAH) accounts for only 3\% of strokes, its profound consequences and unique window for intervention have justified its classification as a separate entity. ${ }^{1}$ Estimated independence varied between $36 \%$ and $60 \%$ only after aSAH. ${ }^{2}{ }^{3}$ Previous studies have suggested that $27-44 \%$ of patients who returned to the community exhibited cognitive dysfunction. ${ }^{4-6}$ In a systemic review of aSAH cognitive dysfunction, the main cognitive domain impairments reported included memory, executive function and language, the prevalence of which ranged from $0 \%$ to $76 \%$ among different studies, although few studies explored the correlations between domain-specific cognitive deficits and disability. ${ }^{3}$ Previous reports confirmed that cognitive deficits were associated with functional outcome at 3 months after aSAH. ${ }^{7}{ }^{8}$ However, they were not adjusted for motor function. There was also suggestion that cognitive dysfunction might further improve even after 9 months. ${ }^{9}$ It would be of interest to understand the pattern and impact of chronic cognitive dysfunction on functional outcomes.

There is another important reason to understand impact of cognitive impairment after aSAH. Currently employed neurological outcome measures may not be disease-sensitive enough to detect aSAH-related brain injuries, and this lack of sensitivity may account for negative results in aSAH neuroprotection trials. Cognitive impairment was thought to be a potential candidate to target in future clinical trials. Thus, there is an urgent need to better understand cognitive impairment after aSAH.

We therefore aimed to (a) describe the cognitive domain deficit pattern at 1 year; (b) evaluate the impact of cognitive domain deficits on functional outcomes at 1 year.

\section{METHODS}

This prospective observational four-center study was carried out in Hong Kong. It is registered at ClinicalTrials.gov of the US National Institutes of Health (NCT01038193) and has been approved by hospital ethics committees. This study conforms to the Declaration of Helsinki, and written informed consent was obtained from all of the participants or their next of kin.

The patient inclusion criteria were: (1) spontaneous SAH with angiography-confirmed aetiology of intracranial aneurysms; (2) hospital admission within $96 \mathrm{~h}$ after ictus; (3) between 21 and 75 years of age; (4) a speaker of Chinese (Cantonese); and (5) informed consent from the patients or their next of kin. The patient exclusion criteria were: (a) a history of previous cerebrovascular or neurological disease other than unruptured intracranial aneurysm; or (b) a history of neurosurgical operation before ictus.

Delayed cerebral infarction due to the delayed cerebral ischaemia (DCI) is defined as a new cerebral infarction identified on CT scans after the exclusion of early (including procedure-related) infarctions. ${ }^{10}$ Early infarction is defined as hypodensity apparent on the post-treatment CT scans at 
around 12-24 h after aneurysm treatment. All of the recruited patients had delayed CT scans of their brains at 2-3 weeks after presentation available for assessment. The diagnosis of cerebral infarction due to DCI was made by site investigators and confirmed by a local neuroradiologist.

\section{ASSESSMENTS}

Assessments were conducted 1 year after ictus by one of the two research assistants (psychology graduates) trained by a postdoctoral research psychologist.

\section{Neuropsychological tests for cognitive domains}

The battery of cognitive assessments had previously been applied in our local Chinese population. ${ }^{11}$ The choice of the battery of cognitive assessments is based on: (a) Previous cognitive studies in local Chinese patients and standard cognitive tests validated in the Cantonese-speaking population; (b) A balanced battery covering verbal and visuospatial memory, attention and working memory, executive functions, psychomotor speed, and language.

\section{A. Verbal memory domain}

1. Hong Kong List Learning Test (HKLLT). ${ }^{12}$ The HKLLT was developed based on the California Verbal Learning Test, which has been used in vascular cognitive impairment studies. It is a verbal learning and memory test that consists of two 16-word lists with three learning trials with immediate recall, 10-min delayed recall, and 30-min delayed recall and recognition for each list. The HKLLT has been validated in both normal and pathological local populations. ${ }^{12}$

B. Visuospatial skill and memory domain

1. The Rey Osterrieth Complex Figure ${ }^{13}$ is a commonly used test for assessing visuospatial construction skills and visuospatial memory.

\section{Attention and working memory domain}

1. Verbal and visual digit span forward and backward from the Chinese Wechsler Memory Scale Third Edition ${ }^{14}$ for the examination of simple attention and working memory. Verbal and visual spans have been used as donor scales for composite psychometric measures.

\section{Executive function and psychomotor speed domain}

1. Symbol-Digit Modalities Test. ${ }^{15}$ This brief, easy-toadminister, timed coding test is a variant of the Digit-Symbol Coding Task in the Wechsler Adult Intelligence Scale-Third edition. Timed coding tasks have been used in studies that involve subjects with suspected vascular cognitive impairment. $^{16}$

2. Color Trails Test (CTT). This test originated from the Trail Making Test (TMT), which is used for the timed assessment of psychomotor speed and executive functions. Using coloured numbers instead of the English alphabet, the CTT is considered to be an acceptable cultural substitute for the original TMT with similar psychometric properties. ${ }^{17}$

3. Animal Fluency. This test requires subjects to generate as many animal names as possible in $1 \mathrm{~min}$. It is a simple timed test that sensitively measures speed and activation, as well as such executive processes as clustering, set-shifting, and retrieval. ${ }^{12} 18$

\section{E. Language domain}

1. Modified Boston Naming Test (mBNT). ${ }^{19}$ The Boston Naming Test is the most frequently used confrontation naming test for assessing language. Here, we have used a validated modified version that contains 15 stimuli appropriate for use in Chinese cultures. ${ }^{19}$

Cognitive domain scores were computed by averaging the $\mathrm{z}$ scores of the respective test measures derived from the established age-matched and education-matched norms. A cognitive domain deficit was defined through a cognitive domain $\mathrm{z}$ score $<-1.65$ (below the fifth percentile). We employed the presence of two or more cognitive domain deficits rather than any cognitive domain deficits as the definition of cognitive impairment as in neuropsychological outcome study of the International Subarachnoid Aneurysm Trial. ${ }^{20}$

\section{Modified Rankin Scale}

The Modified Rankin Scale (mRS) is a valid and clinically relevant instrument that is used to assess recovery (death, disability, and dependence) after stroke and is a commonly used outcome measure in SAH studies. ${ }^{21-24}$ The mRS ranges from 0 (no symptoms) to 6 (death). Unfavourable outcome in survivors is defined as mRS 3-5.

\section{Chinese Lawton IADL Scale}

The Lawton IADL Scale is an appropriate instrument for assessing independent living skills. Items that are assessed include the ability to use the telephone, go shopping, prepare food, do the housekeeping and laundry, secure transportation, be responsible for medications and handle finances. ${ }^{25}$ The Chinese version was previously validated and used. ${ }^{26}$

\section{Geriatric Depressive Scale}

Poststroke depressive symptoms were assessed by the validated Chinese version of the 15 -item version of the Geriatric Depressive Scale (GDS). ${ }^{27} 28$ This scale had been validated to have satisfactory accuracy in detecting poststroke depression in the Chinese population. ${ }^{29}$

\section{Abbreviated National Institute of Health Stroke Scale}

We assessed the motor outcome using Abbreviated National Institute of Health Stroke Scale (aNIHSS) that was previously applied in aSAH clinical trials. ${ }^{30} 31$ The aNIHSS is a measure of the level of facial and limb movement and strength and is composed of the facial palsy section (one score from 0 to 3 ) and the motor section (four separate scores for each limb, from 0 to 4 ). The aNIHSS ranges from 0 (normal) to 19 (no movement).

\section{STATISTICAL ANALYSIS}

The statistical analyses were generated using SPSS for Windows V.15.0 (SPSS Inc., Chicago, Illinois, USA) and MedCalc V.12.2.1.0. Categorical data are given as numbers (percentages) unless otherwise specified; numerical data are given as medians and IQR. A difference with a $\mathrm{p}$ value of less than 0.05 was regarded as statistically significant.

Binary logistic regression analyses were performed to evaluate the predictors of functional outcomes and two or more domain deficits using the enter method, with the F probability of entry set at 0.05 and that of removal set at 0.10 . All analyses passed the goodness of fit tests, which included Omnibus tests of model coefficients and the Hosmer and Lemeshow tests. The ORs with 95\% CIs and the percentage accuracy of classifications (with cut value of 0.500 ) were calculated. Cox and Snell $\mathrm{R}^{2}$ and 
Nagelkerke $\mathrm{R}^{2}$ values provided an indication of the degree of variation in the dependent variable explained by the model (from a minimum value of 0 to a maximum value of approximately 1 ).

The reproducibility of assessments was not assessed because it was not the focus of this study and would have been impractical considering the study's design (possible rehearsal effect and additional inconvenience to patients and their families).

\section{RESULTS}

\section{Patient cohort}

During the 26-month period, 168 aSAH patients, aged 2175 years and admitted within $96 \mathrm{~h}$ of ictus, were recruited and consented to participate during the acute admission. The current cohort was an extension of our previous report cohort of 3 -month outcome. ${ }^{8}$ Eighteen patients died before 1 year follow-up, 30 patients could not cooperate for assessments, and 120 patients completed the assessments at 1 year. The profiles of the recruited patients are shown in table 1. Compared to the patients who completed the assessments, the patients who were not assessed (due to death or not follow commands) were more likely to be of less years of formal education, poor admission World Federation of Neurosurgical Societies grade and have DCI.

Table 1 The recruited patient profiles at 1 year

\begin{tabular}{|c|c|c|c|}
\hline & $\begin{array}{l}\text { Patients not } \\
\text { assessed at } \\
1 \text { year } \\
(n=48)\end{array}$ & $\begin{array}{l}\text { Patients completed } \\
\text { assessments at } \\
1 \text { year } \\
(n=120)\end{array}$ & p Value \\
\hline Age, median (IQR) & $56(48-66)$ & $51(46-61)$ & 0.124 \\
\hline Male (\%) & $25(52)$ & $82(68)$ & 0.089 \\
\hline Hypertension (\%) & $23(48)$ & $41(34)$ & $0.039^{*}$ \\
\hline $\begin{array}{l}\text { Years of school } \\
\text { education, median } \\
\text { (IQR) }\end{array}$ & $8(6-9)$ & $9(6-11)$ & $0.011^{*}$ \\
\hline WFNS grade & & & $<0.001$ ** \\
\hline I & $0(0)$ & $73(61)$ & \\
\hline II & $3(6)$ & $29(24)$ & \\
\hline III & $3(6)$ & $3(3)$ & \\
\hline IV & $18(38)$ & $11(9)$ & \\
\hline V & $23(48)$ & $4(3)$ & \\
\hline Location of aneurysm & & & 0.173 \\
\hline Anterior circulation & $37(77)$ & $104(87)$ & \\
\hline Posterior circulation & $11(23)$ & $16(13)$ & \\
\hline Aneurysm treatment & & & 0.517 \\
\hline Coiling & $17(35)$ & $63(53)$ & \\
\hline Clipping & $19(40)$ & $55(46)$ & \\
\hline $\begin{array}{l}\text { Delayed cerebral } \\
\text { infarction }\end{array}$ & $16(33)$ & $12(10)$ & $<0.001^{* *}$ \\
\hline $\begin{array}{l}\text { Modified Rankin } \\
\text { Scale (1 year) }\end{array}$ & & & $<0.001^{* *}$ \\
\hline 0 & $0(0)$ & $36(30)$ & \\
\hline 1 & $0(0)$ & $21(18)$ & \\
\hline 2 & $0(0)$ & $48(40)$ & \\
\hline 3 & $0(0)$ & $13(11)$ & \\
\hline 4 & $0(0)$ & $1(1)$ & \\
\hline 5 & $20(42)$ & $1(1)$ & \\
\hline 6 & $28(58)$ & $0(0)$ & \\
\hline
\end{tabular}

Cognitive domain deficits

The prevalence of cognitive deficits in the five tested domains ranged from $7 \%$ to $15 \%$ (table 2 ). The highest prevalence was in visuospatial memory and skill, and the lowest prevalence was in verbal memory as well as attention and working memory. No adverse events were reported in relation to the assessments.

\section{Functional outcomes}

At 1 year, unfavourable outcome (mRS 3-5) was independently associated with number of cognitive domain deficits (OR, 2.3; 95\% CI 1.4 to $3.8 ; \mathrm{p}=0.002)$, aNIHSS score (OR, $2.1 ; 95 \% \mathrm{CI}$ 1.1 to $4.0 ; \mathrm{p}=0.021)$, and GDS score (OR, $1.24 ; 95 \%$ CI 1.1 to $1.3 ; \mathrm{p}<0.001$ ), which correctly classified $87.5 \%$ of cases. Unfavourable outcome (mRS 3-5) was also significantly associated with the presence of two or more domain deficits (OR, $8.8 ; 95 \%$ CI 1.9 to $39.4 ; \mathrm{p}=0.005$ ), after adjustments of aNIHSS and GDS scores.

At 1 year, dependent instrumental activity of daily living (IADL $<15)$ was independently associated with number of cognitive domain deficits (OR, 1.8; 95\% CI 1.1 to $2.9 ; \mathrm{p}=0.015)$, aNIHSS score (OR, $1.9 ; 95 \%$ CI 1.0 to $3.5 ; \mathrm{p}=0.038)$, and GDS score (OR, $1.2 ; 95 \%$ CI 1.1 to $1.2 ; \mathrm{p}<0.001$ ), which correctly classified $89.2 \%$ of cases. Dependency (IADL $<15$ ) was also significantly associated with the presence of two or more domain deficits (OR, 4.4; 95\% CI 1.1 to $17.8 ; \mathrm{p}=0.038$ ), after adjustments of aNIHSS and GDS scores.

\section{Risk factors}

The presence of two or more domain deficits was independently associated with age (OR, 1.1; 95\% CI 1.1 to $1.2 ; \mathrm{p}<0.001)$ and delayed cerebral infarction (OR, 6.1; 95\% CI 1.1 to 33.5 ; $\mathrm{p}=0.036$ ), after adjustment for years of school education. Admission World Federation of Neurosurgical Societies Grade and mode of aneurysm treatment was not significantly associated with the presence of two or more domain deficits. The presence of any domain deficit was independently associated with age (OR, 1.06; 95\% CI 1.01 to $1.12 ; \mathrm{p}=0.023$ ), after adjustment for years of school education. Aneurysm location was not a significant risk factor for cognitive impairment. The binary logistic regression model as a whole explained between 17\% (Cox and Snell $\mathrm{R}^{2}$ ) and 32\% (Nagelkerke $\mathrm{R}^{2}$ ) of variance in the presence of cognitive impairment and correctly classified $89.2 \%$ of cases.

\section{DISCUSSION}

We identified cognitive domain deficits in 7 to 15 percent of patients 1 year after an aSAH and $13 \%$ had two or more domain deficits. The commonest deficit (15\%) was in

Table 2 Prevalence of cognitive domain deficits and cognitive impairment

\begin{tabular}{lc}
\hline & At 1 year \\
Completed cognitive and neurological assessments & 120 \\
\hline Verbal memory domain deficit & $8(7)$ \\
Visuospatial skill and memory domain deficit & $18(15)$ \\
Attention and working memory domain deficit & $8(7)$ \\
Executive function and psychomotor speed domain deficit & $11(14)$ \\
Language domain deficit & $9(8)$ \\
$\geq 2$ cognitive domain deficits & $15(13)$ \\
\hline Data in number (\%). &
\end{tabular}


visuospatial memory and skill, which was essential for recording information about one's environment and its spatial orientation, might impact on fall risk, and was related to activity of daily living (Barthel Index) at 3 months. ${ }^{3} 732$ Depending on the timing of assessments and definition, prevalence of visuospatial memory and skill domain deficits ranged from 14\% to $49 \%$ in the literature. ${ }^{3}$ We reported a low $(7 \%)$ verbal memory domain deficit at 1 year as compared to $14 \%$ to $61 \%$ in the literature. ${ }^{3}$ Verbal memory is key to language understanding and communication. The verbal memory finding should be further assessed with future hippocampal volume studies. In contrast to executive function and psychomotor speed domain deficit, attention and working memory domain deficits were less common $(7 \%)$ than previously reported due to the more stringent criteria than reported cut-off scores in the tests employed (verbal and visual digit span forward and backward from the Chinese Wechsler Memory Scale). ${ }^{4}$ Our executive function and psychomotor speed domain and attention and working memory domain are considered by some authors as a broader category of executive function or cognitive 'control' (planning and organisation).

Upon systemic review of clinical prediction models for aSAH, it was noted that neither Glasgow Outcome Scale nor mRS were developed for use in $\mathrm{SAH}^{33}$ and their lack of sensitivity might explain the failure to show a benefit from the treatment of angiographic vasospasm and other interventions. ${ }^{34}$ Cognitive outcome measures, in conjunction with other outcome scales, would improve outcome assessments in SAH trials.

We showed that the presence of two or more cognitive domain deficits was associated with disability, as measured by mRS, and with IADL, as measured by Lawton IADL. Stroke-related brain injury often results in a combination of motor and cognitive impairments, and the presence of depression may impact on functional outcome. Although aNIHSS and GDS scores might not completely discriminate the effects or presence of any motor impairment or depression, we demonstrated that the impact of two or more cognitive domain deficits on functional outcomes was independent of aNIHSS and GDS scores. Whether cognitive rehabilitation and medical treatment such as cholinesterase inhibitors could improve cognitive function and neurological outcome should be further investigated. ${ }^{35}$

The findings of the current 1 year study cannot be directly compared with the 3 month Montreal Cognitive Assessment (MoCA) study results, as the definitions of cognitive impairment were different. ${ }^{8}$ In the 3 month MoCA study, the objective is to investigate for alternations in cut-off scores to improve sensitivity and specificity to predict functional outcome. Milder forms of cognitive impairment are less likely to be detected by the MoCA cut-off score $21 / 22 .{ }^{36}$ In the current 1 year outcome study, we employed average z-scores below 1.65 in two or more domains as cognitive impairment. Both studies pointed to the significance of cognitive impairment to functional and neurological outcome. Nevertheless, one must understand that cognitive impairment in relative isolation is devastating in working age people and mRS type outcome scales do not detect this important patient subgroup. Confidently identifying them with comprehensive cognitive testing, or better still, bedside or clinics screening is where further work is needed.

As in normal aging and neurodegenerative diseases, age was shown to be the consistent predictor of presence of any cognitive domain deficit at 1 year. Delayed cerebral infarction was the other important predictor of presence of two or more cognitive domain deficits, supporting the recommendation that delayed cerebral infarction be included as a therapeutic target for clinical trials targeting neuroprotection after aSAH. ${ }^{10}$ Our findings also echoed that presence of two or more domain deficits was a more sensitive cognitive outcome measure than presence of any domain deficit. ${ }^{20}$ Delayed cerebral infarction was shown to predict unfavourable outcome in terms of Extended Glasgow Outcome Scale, poor outcome in terms of mRS, and dependent activity of daily living in terms of Barthel Index at 6 months, after adjustments for other prognostic factors. ${ }^{37}$ In a recent meta-analysis, delayed cerebral infarction was predicted by haemorrhage load, clinical condition on admission, and other risk factors such as smoking. ${ }^{38}$ These data further supported the importance to target delayed cerebral infarction in future researches.

\section{Limitations of this study}

This study has several limitations. First, despite the fact that the neuropsychological battery was validated in the Chinese population with established norms, it might not be sensitive and comprehensive enough to detect all patients with cognitive impairment. ${ }^{39}$ Self-reported cognitive impairment was not assessed in the current study. Second, the functional and cognitive domain outcomes are dichotomised for analyses rather than using the weighted continuous scores. The clarity in understanding the meaning of the categories may reduce the chance of detecting statistical differences. Third, we employed self-report of IADL instead of performance-based assessment. Although self-reporting facilitated the inclusion into the current battery of assessments, reliance of self-report may be biased by depressive symptoms. ${ }^{40}$ Fourth, aNIHSS and GDS scores might not completely discriminate the effects or presence of any motor impairment or depression. Fifth, although the whole battery of assessments was not validated for aSAH patients, individual tests were validated and applicable to our local population. Sixth, early cerebral infarction and their causes were not included in the design of the current multicenter study. They should be considered in future study. Lastly, the battery of cognitive and functional assessments is tiring, although we offered a 5-10 min rest in the middle of the assessment sessions or divided the assessments between two consecutive days. Fatigue was not assessed during testing.

\section{CONCLUSIONS}

We were able to show that (1) Cognitive domain deficits occurred in $7 \%$ to $15 \%$ of the cohort and correlated with the disabilities and dependent instrumental activities of daily living at 1 year, independent of motor and depression scores; (2) Delayed cerebral infarction was an independent risk factor of cognitive impairment at 1 year.

\section{Acknowledgements None.}

Collaborators Cognitive Dysfunction after Aneurysmal Subarachnoid Hemorrhage Site Investigators: Prince of Wales Hospital: GKCW, WSP and VM; Kwong Wah Hospital: John Kwok, Kwong Yau Chan, Peter Woo, Calvin Mak and Peter Pang; Princess Margaret Hospital: Yin Chung Po, Tony Chan, Wai Kei Wong and Simon Lee; Pamela Youde Nethersole Eastern Hospital: Chi Keung Wong, Michael Lee, Rebecca Ng, Alain Wong and Vincent Pang.

Contributors GKCW, AW, and VM conceived and designed the study; Cognitive Dysfunction after Aneurysmal Subarachnoid Hemorrhage Investigators were responsible for patient recruitment; SWL and KN assessed the patients; GKCW and WSP supervised the study; GKCW drafted the manuscript; all authors critically reviewed and agreed to the final version of the manuscript.

Funding This study was supported by the Neurosurgery Research and Training Fund, the Chinese University of Hong Kong.

Competing interests None.

Ethics approval Joint NTEC-CUHK Clinical Ethics Committee.

Provenance and peer review Not commissioned; externally peer reviewed. 
Data sharing statement The data could be accessed with the corresponding authors upon agreement for collaboration.

Open Access This is an Open Access article distributed in accordance with the Creative Commons Attribution Non Commercial (CC BY-NC 3.0) license, which permits others to distribute, remix, adapt, build upon this work non-commercially, and license their derivative works on different terms, provided the original work is properly cited and the use is non-commercial. See: http://creativecommons.org/licenses/by-nc/3.0/

\section{REFERENCES}

1 Chau PH, Woo J, Goggins WB, et al. Trends in stroke incidence in Hong Kong differ by stroke subtype. Cerebrovasc Dis 2011:31:138-46.

2 Rinkel GJ, Algra A. Long-term outcomes of patients with aneurysmal subarachnoid haemorrhage. Lancet Neurol 2011:10:349-56.

3 Al-Khindi T, Macdonald RL, Schweizer TA. Cognitive and functional outcome after aneurysmal subarachnoid hemorrhage. Stroke 2010:41:e519-36.

4 Wong GK, Wong R, Mok VC, et al. Clinical study on cognitive dysfunction after spontaneous subarachnoid haemorrhage: patient profiles and relationship to cholinergic dysfunction. Acta Neurochir 2009;151:1601-7.

5 Wong GK, Wong A, Mok V, et al. Natural history and medical treatment of cognitive dysfunction after spontaneous subarachnoid haemorrhage: review of current literature with respect to aneurysm treatment. J Neurol Sci 2010;299:5-8.

6 Schweizer TA, Al-Khindi T, Macdonald RL. Mini-mental state examination versus Montreal cognitive assessment: rapid assessment tools for cognitive and functional outcome after aneurysmal subarachnoid hemorrhage. J Neurol Sci 2012;316:137-40.

7 Mayer SA, Kreiter KT, Copeland D, et al. Global and domain-specific cognitive impairment and outcome after subarachnoid hemorrhage. Neurology 2002:59:1750-8.

8 Wong GK, Lam S, Ngai K, et al. Evaluation of cognitive impairment by the Montreal Cognitive Assessment in patients with aneurysmal subarachnoid hemorrhage: prevalence, risk factors, and correlations with 3-month outcomes. J Neurol Neurosurg Psychiatry 2012;83:1112-17.

9 Powell J, Kitchen N, Heslin J, et al. Psychosocial outcomes at 18 months after good neurological recovery from aneurysmal subarachnoid haemorrhage. J Neurol Neurosurg Psychiatry 2004;75:1119-24.

10 Vergouwen MD, Vermeulen M, van Gijn J, et al. Definition of delayed cerebral ischemia after aneurysmal subarachnoid hemorrhage as an outcome event in clinical trials and observational studies. Proposal of a multidisciplinary research group. Stroke 2010;41:2391-5

11 Wong GK, Ngai K, Wong A, et al. Long-term cognitive dysfunction in patients with traumatic subarachnoid hemorrhage: prevalence and risk factors. Acta Neurochir 2012:154:105-11.

12 Chan AS, Kwok I. Hong Kong list learning test: manual and preliminary norm. Hong Kong: Department of Psychological and Clinical Psychology Center, 1999.

13 Wechsler D. Wechsler memory scale III (Chinese): manual. Taiwan: Psychological Corporation, 2005.

14 Rey A. L'examen psychologique dans les cas d'encephalopathie traumatique. Arch de Psychologie 1941;28:296-302.

15 Smith A. Symbol Digit Modalities Test (SDMT) manual. Revised edn. Los Angeles: Western Psychological Services, 1982

16 O'Sullivan M, Morris RG, Markus HS. Brief cognitive assessment for patients with cerebral small vessel disease. J Neurol Neurosurg Psychiatry 2005;76:1140-5.

17 Lee TM, Chan CC. Are trail making and color trails tests of equivalent constructs? J Clin Exp Neuropsychol 2000;22:529-34.

18 Chan A, Ho S, Poon WS. Neuropsychological sequalae of patients treated with microsurgical clipping or endovascular embolization for anterior communicating artery aneurysm. Eur Neurol 2002;47:37-44

19 Tham W, Auchus AP, Thong M, et al. Progression of cognitive impairment after stroke: one year results from a longitudinal study of Singaporean stroke patients. J Neurol Sci 2002;203-204:49-52.
20 Scott RB, Eccles F, Molyneux AJ, et al. Improved cognitive outcomes with endovascular coiling of ruptured intracranial aneurysms: Neuropsychological outcomes from the International Subarachnoid Aneurysm Trial. Stroke 2010:41:1743-7.

21 Rankin L. Cerebral vascular accidents in patients over the age of 60. II. Prognosis. Scott Med J 1957;2:200-15

22 van Sieten JC, Koudstaal PJ, Visser MC, et al. Interobserver agreement for the assessment of handicap in stroke patients. Stroke 1988;19:604-7.

23 Banks JL, Marotta CA. Outcomes validity and reliability of the modified Rankin Scale: implications for stroke clinical trials: a literature review and synthesis. Stroke 2007;38:1091-6.

24 Wong GK, Poon WS, Boet $\mathrm{R}$, et al. Intravenous magnesium sulphate after aneurysmal subarachnoid hemorrhage: a multi-center phase III study. Stroke 2010;41:921-6.

25 Lawton MP, Brody EM. Assessment of older people: self-maintaining and instrumental activities of daily living. Gerontologist 1969;9:179-86.

26 Tong A, Man DW. The validation of the Hong Kong Chinese version of the Lawton Instrumental Activities of Daily Living Scale for institutionalized elderly persons. OTJR: Occup Particip Health 2002;22:132-42.

27 Brink TL, Yesavage JA, Lum 0 , et al. Screening tests for geriatric depression. Clin Gerontol 1982:10:37-42.

28 Lim PP, Ng LL, Chiam PC, et al. Validation and comparison of three brief depression scales in an elderly Chinese population. Int J Geriatr Psychiatry 2000;15:824-30.

29 Tang WK, Mok V, Chan SS, et al. Screening of dementia in stroke patients with lacunar infarcts: comparison of the Mattis dementia rating scale and the mini-mental state examination. J Geriatr Psychiatry Neurol 2005;18:3-7.

30 Macdonald RL, Higashida RT, Keller E, et al. Clazosentan, an endothelin receptor antagonist, in patients with aneurysmal subarachnoid haemorrhage undergoing surgical clipping: a randomised, double-blind, placebo-controlled phase 3 trial. Lancet Neurol 2011;10:618-25

31 Macdonald RL, Higashida RT, Keller E, et al. Randomized trial of clazosentan in patients with aneurysmal subarachnoid hemorrhage undergoing endovascular coiling. Stroke 2012:43:1463-9.

32 Rapport LJ, Dutra RL, Webster JS, et al. Hemispatial deficits on the Rey-Osterrich Complex Figure drawing. Clin Neuropsychol 1995;9:169-79.

33 Jaja BN, Cusimano MD, Etminan N, et al. Clinical prediction models for aneurysmal subarachnoid hemorrhage: a systemic review. Neurocrit Care 2013:18:143-53.

34 Macdonald RL, Michael CD, Etminan N, et al. Subarachnoid Hemorrhage International Trialists Data Repository (SAHIT). World Neurosurg 2013. doi:10.1016/ j.wneu.2013.01.006

35 Wong GK, Wong R, Mok V, et al. Rivastigmine for cognitive impairment after spontaneous subarachnoid haemorrhage: a pilot study. J Clin Pharm Ther 2009:34:1-7

36 Wong A, Xiong YX, Wang D, et al. The NINDS-Canadian stroke network vascular cognitive impairment neuropsychology protocols in Chinese. J Neurol Neurosurg Psychiatry 2013;84:499-504.

37 Wong GK, Poon WS. Clinical, transcranial Doppler ultrasound, radiological features, and prognostic significance of delayed cerebral ischemia. Acta Neurochir Suppl 2013:115:9-11.

38 de Rooij NK, Rinkel GJ, Dankbaar JW, et al. Delayed cerebral ischemia after subarachnoid hemorrhage. A systemic review of clinical, laboratory, and radiological predictors. Stroke 2013;44:43-54

39 Scott RB, Eccles F, Lloyd A, et al. From multidimensional neuropsychological outcomes to a cognitive complication rate: the International Aneurysm Trial. Trials 2008;9:13.

40 Blackstone K, Moore DJ, Heaton RK, et al. Diagnosing symptomatic HIV-associated neurocognitive disorders: self-report versus performance-based assessment of everyday functioning. J Int Neuropsychol Soc 2012;18:79-88. 